\title{
Advanced Instrumentation for Polyharmonic Metal Detectors
}

\author{
Jakub Svatoš ${ }^{1}$, Josef Vedral ${ }^{1}$, Tomas Pospisil ${ }^{1}$ \\ ${ }^{1}$ Czech Technical University in Prague, Prague 6, Technicka 2, 166 27, Czech Republic
}

\begin{abstract}
The paper describes application of polyharmonic sinc signals in devices for metal object detection. A basic problem of conventional eddy current metal detectors (induction devices in general) lies in its limited possibilities of discrimination or identification of detected objects. To better characterize the detected object, the excitation signal and the following signal processing in the detector should be done. Application of polyharmonic excitation signal and its processing could bring an opportunity to improve the determination ability. The article is focused to the polyharmonic sinc signal. Spheres of different diameters and from different ferrous and non-ferrous materials were used as specimens. Experimental measurements were processed in frequency domain - amplitude and phase spectra were calculated and presented. As part of the work, classifications of ferrous and non-ferrous materials were done based of measured data as well as classification of individual ferrous including non-ferrous materials and estimation of the size of the classified object. Support vector classifier was primarily used for data classification.
\end{abstract}

\section{Index Terms - Classification, Eddy currents, Metal identification, Polyharmonic signal, Signal processing}

\section{INTRODUCTION}

$\mathrm{M}$ etal detectors are nowadays used in industry, metals separation/distinguishing, detection of metal contamination in food and medicine products, detection of dangerous metal objects in security, archelogy or humanitarian demining, unexploded ordnance detection etc. [1]-[4]. Electromagnetic detectors, which are still most commonly used type of metal detectors, due to low cost, have essential shortcoming: these detectors poorly recognize/discriminate different type of materials (with similar electromagnetic properties - conductivity and permeability) or objects (e.g. mines). It is due to the fact that the information about material of metallic objects are mainly contained in phase shift of the received signal (response) [5]. For standard single tone methods it leads to limited discrimination ability. It is caused by the fact that some objects have similar response (e.g. gold and tinfoil) [6]. Other methods, e.g. Ground Penetrating Radar have better discrimination capabilities, but they are expensive [7].

In this paper we present a novel approach based on polyharmonic excitation signal which shows better discrimination ability for electromagnetic detectors. Application of polyharmonic sinc signal and its processing could bring an opportunity to improve the discrimination ability. Thanks to multiple frequencies of a sinc signal, a response of detected object is measured in a wide band. In the response, information about detected object are carried by multiple phases and amplitudes. More detailed information about a detected object than by using classical single tone methods is obtained. Experimental results has verified that application of a sinc signal can bring more accurate identification of objects than by using classical methods.

\section{Methods}

On the basis of previous research (published in [8] and [9]), this article brings new approach based on difference between spectrum of signal which corresponds to measurement without any object (background) and spectrum of signal which correspond to measurement with a specific testing sample. By this way of signal processing there is no need to control the change of impedance of the excitation coil for each frequency. This spectrum difference is used for further classification.

All measurements were taken on search head of metal detector ATMID [10]. As an excitation signal voltage sinc signal were used. Its main advantage include that all frequencies are applied at once and thanks to multiplefrequencies, a wide range of the Response Function of the detected object is measured. More details about Response Function can be found in [11] or [12]. Also signal parameters (start and stop frequency, number and position of spectral lines) are very easily defined. Signal can by defined as antisymmetric, allows to cover whole input range of analogue-todigital converter (peak-to-peak amplitude) and to continuous crossing between periods.

A modified voltage excitation sinc signal, which is used for experiment, is composed of two sinc signals with the same parameters where the second half of the period is inverted (Fig. 1). One period of the signal is described by the formula:

$$
u(t)=H\left(t+\frac{T_{1}}{2}\right)\left[\frac{\sin \left(\frac{2 \cdot \pi \cdot t}{T_{2}}\right)}{\frac{2 \cdot \pi \cdot t}{T_{2}}}\right]-H\left(t-\frac{T_{1}}{2}\right)\left[\frac{\sin \left(\frac{2 \cdot \pi \cdot t}{T_{2}}\right)}{\frac{2 \cdot \pi \cdot t}{T_{2}}}\right],
$$

where $H$ is Heaviside function, which chops the segment of the sinc function in time range $\left.<-T_{1 / 2}, T_{1 / 2}\right), T_{1}=1 / f_{1}$ corresponds to start/lowest frequency and $T_{2}=1 / f_{2}$ corresponds to stop/highest frequency (see Fig. 1) and $t$ is time. The number of spectral lines is given by ratio of the $T_{1}$ and $T_{2}$. Since the sinc signal is composed of all frequencies equally its spectrum has rectangular shape with equidistantly distributed frequencies.

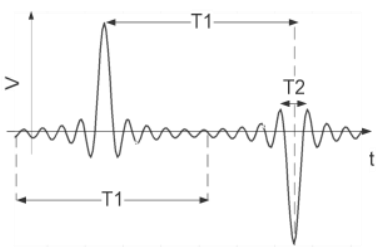

Fig. 1. Time plot of the modified sinc signal

The following parameters describe the used modified sinc signal, frequency $f_{1}=1 / T_{1}=1 \mathrm{kHz}, f_{2}=1 / T_{2}=10 \mathrm{kHz}$, 
10 significant carrier frequencies, and amplitude of $10 \mathrm{~V}$. From this parameters repeating frequency of $f_{\mathrm{R}}=1 \mathrm{kHz}$ follows and significant frequencies at each $2 \mathrm{kHz}$. Since the coil is excited by current $i_{\mathrm{L}}$, its voltage $u_{\mathrm{L}}$ correspond to the first derivative.

Block diagram of the measurement setup is in Fig. 2. The generator AFG 3102 was externally synchronized from the synchronizing output of the digitizer. The 15-bit digitizer was triggered by TTL signal from the generator. This is done to ensure the repeatability of the sinc signal generation.

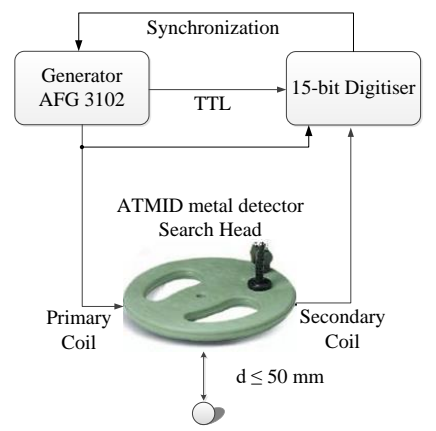

Fig. 2. Block diagram of the measurement setup

The ATMID search head uses a Double-D coils configuration with a diameter of $260 \mathrm{~mm}$. Number of turns of transmitting coil is 17 and receiving coil is approx. 190 turns. The frequency range of used sinc signal was below the resonant frequency of the both coils (transmitting coil about $90 \mathrm{kHz}$ receiving coil about $45 \mathrm{kHz}$ ).

In total, five different materials represented by spheres of different diameters were measured as samples. Three nonferromagnetic materials (bronze, brass and stainless steel AISI 316 spheres) and two ferromagnetic materials (stainless steel AISI 420 and chrome steel AISI 52100 100Cr6 spheres). Stainless steel AISI 316 spheres represents material with relative permeability slightly larger than one; $\mu_{\mathrm{r}}=1.02$. All materials were measured for different diameters of spheres (diameter of $d=10 \mathrm{~mm}, 13 \mathrm{~mm}, 15 \mathrm{~mm}, 20 \mathrm{~mm}, 22 \mathrm{~mm}$ and $25 \mathrm{~mm}$ ). Spheres were placed to maximal distance of $50 \mathrm{~mm}$ from the coil in the axis of greatest sensitivity.

\section{RESULTS}

Samples were placed in open air. The excitation signal was driven through the transmitting coil of ATMID metal detector. Measured data were digitized and processed in MATLAB.

First, a background signal without the presence of any tested objects is measured. Next, this signal is compared with the signals corresponding to the tested spheres. The all voltage signals were filtered by Finite Impulse Response (FIR) bandpass filter of 200th order. Lower frequency of the band pass filter was $f_{\mathrm{d}}=1 \mathrm{kHz}$ and upper frequency $f_{\mathrm{h}}=30 \mathrm{kHz}$. Filtered signal was after that processed by Discrete Fourier Transform (DFT) using a Fast Fourier Transform (FFT) algorithm, and were computed from number of samples $\mathrm{N}=1048578$, which is nearest powers of two from 1 MSamples. The phase spectra were calculated from a complex variable definition. All presented phase spectra were computed as a difference between the phase spectrum of the signal which corresponds to measurement without any object (background) and the phase spectrum of the signal which correspond to measurement with a specific testing sample. For better presentation and comparison, the spectra of comparing signals which are presented are shifted by $\pm 100 \mathrm{~Hz}$ in the figures.

Fig. 3 shows example of the measured amplitude and phase spectra which corresponds to the ferromagnetic AISI 52100 $100 \mathrm{Cr} 6$ testing spheres. By comparing of four different specimen with different diameters $(d=10 \mathrm{~mm}, 15 \mathrm{~mm} 20 \mathrm{~mm}$ and $25 \mathrm{~mm}$ ) from ferrous chrome steel $100 \mathrm{Cr} 6$ it is evident that with increasing diameter the induced voltage increases uniformly. Differences between induced voltages getting smaller on higher frequencies this can be explained by approaching the Inductive limit of the Response Function (see [6] or [12]). Phase spectra show differences in phases for individual the spheres. The difference increases with various diameters at higher frequencies especially. Spheres of larger diameter changes phase more than those of smaller diameter.
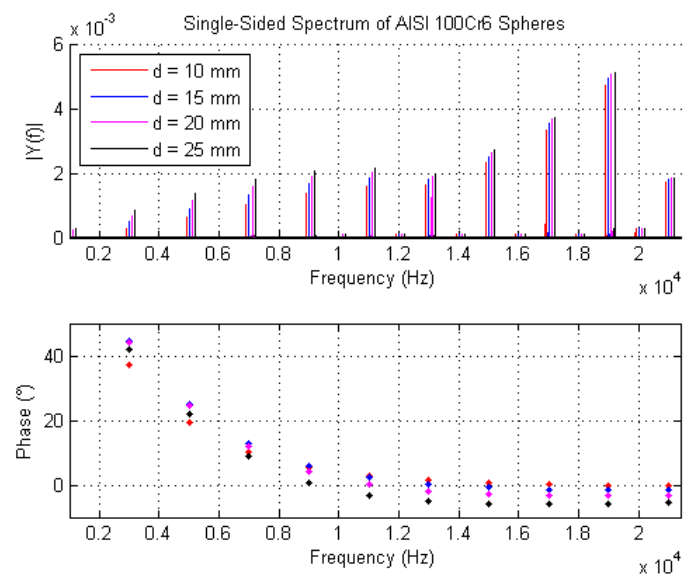

Fig. 3. Amplitude spectra and Phase spectra corresponding to AISI 52100 $100 \mathrm{Cr} 6$ with diameters of $10 \mathrm{~mm}, 15 \mathrm{~mm}, 20 \mathrm{~mm}$ and $25 \mathrm{~mm}$

The results of comparison of four different bronze spheres (diameters $d=10 \mathrm{~mm}, 13 \mathrm{~mm}, 20 \mathrm{~mm}$ and $22 \mathrm{~mm}$ ) from a nonferrous material is shown in Fig. 4.
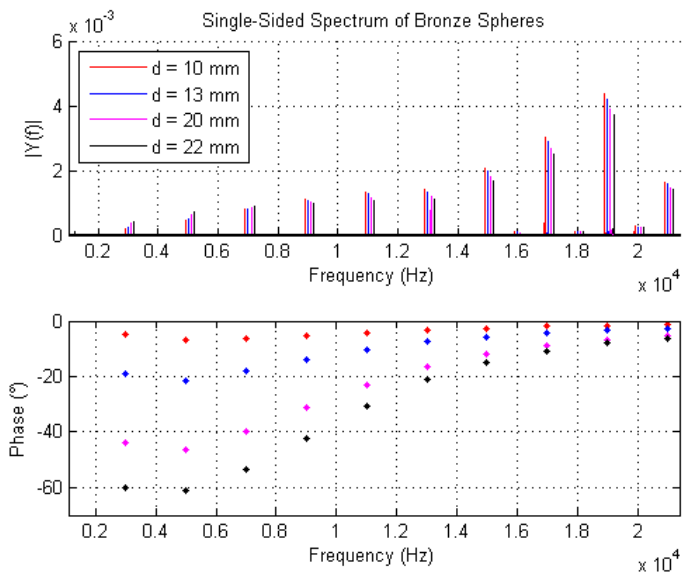

Fig. 4. Amplitude spectra and Phase spectra corresponding to bronze sphere with diameters of $10 \mathrm{~mm}, 15 \mathrm{~mm}, 20 \mathrm{~mm}$ and $25 \mathrm{~mm}$

The change of induced voltage has opposite trend, with increasing diameter of the sphere induced voltage decreases uniformly. It can be explained by negative phase shift. It is known [6] that non-ferrous materials change phase to negative values only. At lower frequencies absolute phase shift difference increasing greatly with diameter and getting smaller 
at higher frequencies thanks to character of the Response Function. This phase shift caused by non-ferrous is in contrast with ferrous materials. Phase shift, caused by different sizes of ferrous material, changes a little at lower frequencies.

The measurement proves an apparent difference between ferrous and non-ferrous materials. Ferrous materials cause phase shift from positive to negative values and significant signal magnification at all carrier frequencies. Non-ferrous objects cause negative phase shift and signal diminution at higher frequencies.

A closer comparison of similar materials is presented on the next Figs 5 - 7. Since induced voltage is highly dependent on the position of the sample, it is important for comparison of similar materials to keep the location of samples at the same position. All compared results were measured in the exactly defined position: in highest axis of sensitivity of the detector head at a distance of $50 \mathrm{~mm}$ from the detector.
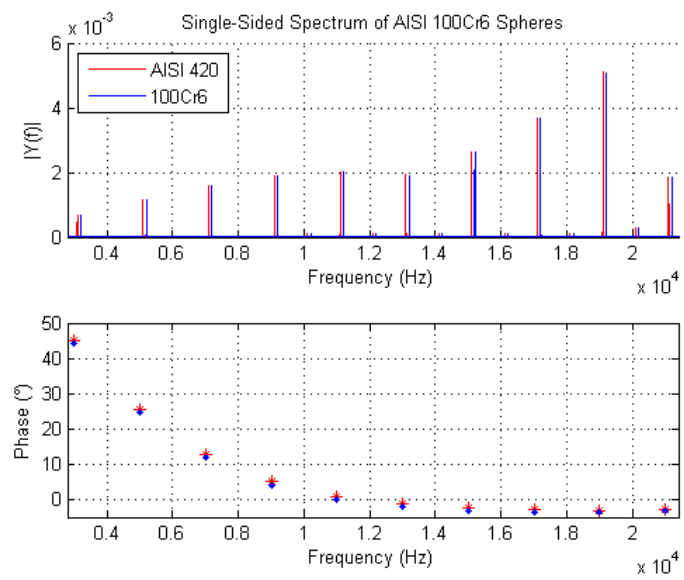

Fig. 5. Amplitude spectra, Phase spectra corresponding to AISI 420 and AISI $52100100 \mathrm{Cr} 6$ sphere with diameters of $20 \mathrm{~mm}$

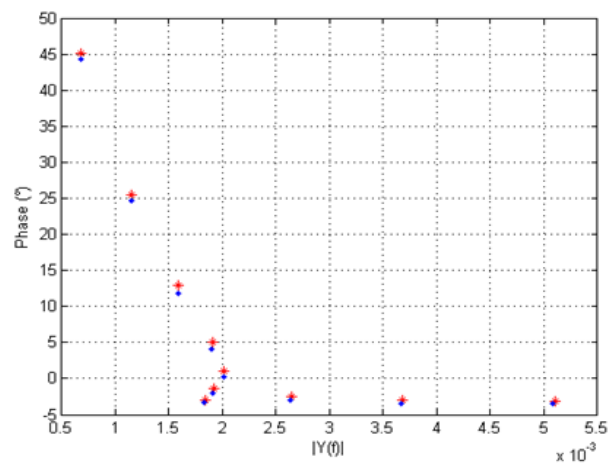

Fig. 6. X-Y chart corresponding to AISI 420 (red) and AISI $52100100 \mathrm{Cr} 6$ (blue) sphere with diameters of $20 \mathrm{~mm}$

Fig. 5 shows amplitude and phase spectra concerning two specimen from similar materials; INOX AISI 420 and AISI $52100100 \mathrm{Cr} 6$ sphere with diameters of $20 \mathrm{~mm}$. Phase difference corresponding to these materials is slightly larger than 1 degree at lower frequencies and slightly smaller than 1 degree at higher frequencies; difference between both materials is in the trend of the phase spectrum. Difference between the amplitude spectra is also minimal. The biggest difference is at the $10^{\text {th }}$ harmonic. For better viewing (Fig. 6) X-Y chart is also presented (x-axis $\sim$ magnitude, $\mathrm{y}$-axis $\sim$ phase).

When comparing non-ferrous materials with similar electromagnetic properties (brass and bronze) similar situation occurs. Comparison of spheres with diameter of $d=20 \mathrm{~mm}$ is shown in X-Y chart (Fig. 7). The difference in the amplitude spectrum increases with increasing frequency. The phase spectra difference decreases with increasing frequency.

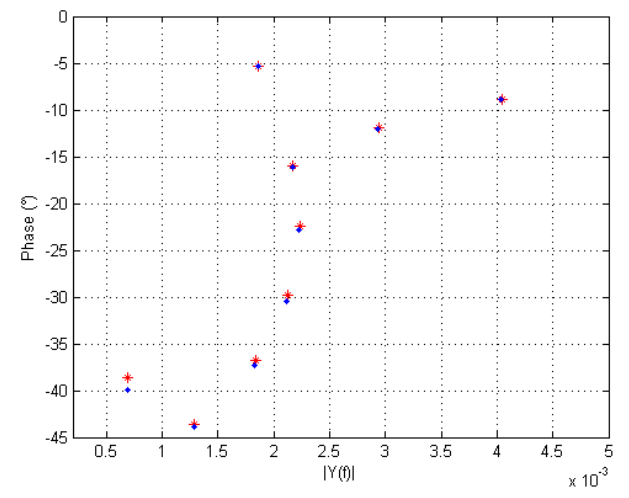

Fig. 5.40: $\mathrm{X}-\mathrm{Y}$ chart corresponding to brass (red) and bronze (blue) sphere with diameters of $20 \mathrm{~mm}$

Phase difference at lower frequencies is about 1.5 degree for lower frequencies and only about 0.2 degree for higher frequencies. The trend of the phase shift depends on response parameter $\alpha$ [6]. Response parameter $\alpha$ of brass is approx. twice as bigger as of bronze. It leads to a smaller change in phase.

Based on the experimental results, use of sinc excitation signals allows possible identification and discrimination of the detected objects. The application of polyharmonic excitation signal bring an opportunity for better response in a wide range of frequencies, and therefore more extensive and complex set of data for the signal analysis is available. It is evident that thanks to the Response Function the discrimination between different materials by means of classifier can be done.

All data processing and classification was done using MATLAB software with classification pattern recognition toolbox PRTools (version 5) [13]. The data obtained from individual measured samples were digitized, processed (as described above) and stored as dataset structure in MATLAB. These datasets were used for further classification process. In total 80 sets of repeated measurements, represented by its datasets, were taken for classification. One measurement set (dataset) contains 20 individual measured samples. Measured materials are Brass, Bronze, INOX AISI 316 and AISI 420 and $52100100 \mathrm{Cr} 6$ of diameters $d=10 \mathrm{~mm}, 13 \mathrm{~mm}, 15 \mathrm{~mm}, 20 \mathrm{~mm}$, $22 \mathrm{~mm}$ and $25 \mathrm{~mm}$. All repeatedly measured samples were saved to nested structure. As written above nested structure consists of 80 sets of different measurement, where each set of measurement contains same 20 individual samples. As only amplitude and phase spectra at desired frequencies are relevant, the algorithm for maximum peak detection near significant frequencies was applied. Thanks to that a set of 10 desired frequencies and corresponding 10 values of amplitude spectra and 10 values of phase spectra difference (as mentioned above, phase spectra were computed as the difference between signal with and without sample) for each sphere and every measurement is obtained. This gives a 20-dimensional space for classification. To classify into individual materials classes, more complex approach and additional features must be 
considered. Thanks to similar responses new features have to be found. To improve the classification, ratio of magnitudes and phases of each 10 investigating frequency were added together with the differences between two subsequent values of phases (9 features). This adds 19 new features and together with 20 basic features it gives 39 dimensional space.

For classification Support Vector Machine (SVM) classifier with linear kernel [14] was used, for comparison Naive Bayes classifier is also presented. Both classifiers were trained on training data ( 24 from 80 datasets) and tested on remaining data (56 from 80 dataset). The size of training data was selected using Learning curve [15].

Results of basic classification into two main classes - ferrous and non-ferrous materials for experimental measured datasets are displayed in Table 1.

TABLE 1

Comparison of two classifiers and its success rate in \% into ferrous $\mathrm{x}$ nonferrous classes

\begin{tabular}{|c|c|c|}
\hline Classifier & SVM & Naive Bayes \\
\hline Polyharmonic approach & $100 \%$ & $97.3 \%$ \\
\hline Classical single tone method & $96.9 \%$ & $95.7 \%$ \\
\hline
\end{tabular}

From these classifying results it is evident that if the multiple frequency approach is used ( 20 dimensional space), there is $100 \%$ chance to differentiate between ferrous and non-ferrous materials with comparison to classical single tone methods where classification success drops to $96.9 \%$.

Classification into individual ferrous and non-ferrous materials present Table 2 and 3 .

TABLE 2

Comparison of two classifiers and its success rate in $\%$ into individual non-ferrous classes

\begin{tabular}{|c|c|c|}
\hline Classifier & SVC & Naive Bayes \\
\hline Polyharmonic approach & $91.3 \%$ & $90 \%$ \\
\hline Classical single tone method & $68.2 \%$ & $66.3 \%$ \\
\hline
\end{tabular}

From Table 2 is evident that if classical single tone method is used, classifying success of SVC into individual non-ferrous materials is less than $70 \%$ but if polyharmonic approach is applied classification success increases to more than $91 \%$ when all 39 features are used.

Similar results (Table 3) gives classification into individual ferrous materials.

TABLE 3

Comparison of two classifiers and its success rate in \% into individual ferrous classes

\begin{tabular}{|c|c|c|}
\hline Classifier & SVC & Naive Bayes \\
\hline Polyharmonic approach & $97.3 \%$ & $86.1 \%$ \\
\hline Classical single tone method & $66.9 \%$ & $78.3 \%$ \\
\hline
\end{tabular}

If single tone method (two features - one frequency and one phase) is used, SVC success rate was only $66.9 \%$. The success rate increased to $97.3 \%$, when all 39 features for classification is used. This confirms previous results that if multiple frequencies are used, there are more features for classification (39) available and therefor better results can be achieved.

Experiment showed that if multiple frequencies - their magnitudes and phases are used as features for classification and new features (relations between existing ones) are added, more successfully classification with comparison to classical single tone methods can be done.

\section{CONCLUSION}

The main shortcoming of present day detectors and their identification ability lies in the fact that the majority of them use only single frequency for detecting and identifying objects. The polyharmonic sinc signal has been shown as appropriate for further spectral analysis of the Response Function. Thanks to amplitude and phase spectra with multiple frequencies the Response Function of the detected object was measured over a wide band of frequencies. Measured data showed that if multiple frequencies (its magnitudes and phases) are used as features and new features (relations between existing ones) are added, the classification can be done more successfully with comparison to classical single tone methods.

\section{ACKNOWLEDGMENT}

This research was supported by the Grants SGS15/153/OHK3/2T/13 "Systems of Data Processing and Fusion for Diagnostics and Measurement Applications" by the Grant Agency of the Czech Technical University in Prague.

\section{REFERENCES}

[1] Z. Bielecki, J. Janucki, et al., "Sensors and Systems for the Detection of Explosive Devices - An Overview," Metrology and Measurement Systems, vol. XIX, no. 1, pp. 3-28, 2012.

[2] H. Krüger, H. Ewald, "Handheld metal detector with online visualisation and classification for the humanitarian mine clearance", IEEE SENSORS 2008, pp. 415-418, 2008.

[3] L.F. Stine, D.L. Shumate, "Metal detecting: An effective tool for archaeological research and community engagement", North American Archaeologist, 36 (4), pp. 289-323, 2015.

[4] Y. Zhao, W. Yin, et al., "On the low-frequency electromagnetic responses of in-line metal detectors to metal contaminants", IEEE Transactions on Instrumentation and Measurement, vol. 63, no. 12, pp. 3181-3189, 2014.

[5] C. Bruschini, A Multidisciplinary Analysis of Frequency Domain Metal Detectors for Humanitarian Deminig, Ph. D. dissertation thesis, Vrije Universiteit Brusse, 2002.

[6] Composite Autors, Metal Detector Basic and Theory, Fortress Technology Inc, 1997.

[7] R. C. Doheny, S. Burke, R. Cresci, P. Ngan, R. Walls, Handheld Standoff Mine Detection System (HSTAMIDS) Field Evaluation in Thailand, Assistant Secretary of Defense, (Special Operations and Low - Intensity Conflict), 2008.

[8] J. Svatos, J. Vedral, P. Novacek, "Metal Object Detection and Discrimination Using Sinc Signal," BEC 2012 - Proceedings of the 13th Biennial Baltic Electronics Conference, Tallinn Technical University, pp. 307-310, 2012.

[9] J. Svatos, J. Vedral, P. Novacek, "Sin(x)/x Signal Utilization in Metal Detection and Discrimination," Magnetic Measurements 2012, Bratislava, Slovak University of Technology, pp. 46, 2012.

[10] Schiebel. ATMID Maintenance Manual MT5001/16/010E.

[11] L. S. Riggs, J., E. Mooney, "Identification of Metallic Mine-Like Objects Using Low Frequency Magnetic Fields," IEEE Transactions on Geoscience \& Remote Sensing, vol. 39, no. 1, 2001.

[12] J. Svatos, P. Fexa, J. Vedral, "Metal Detector Excited by Frequency Swept signal," Metrology and Measurement Systems, vol. 18, no. 1, p. 57-68, 2011.

[13] Delft University of Technology, 2014. [online] Available at <http://prtools.org/>.

[14] F. Heijden, R. P. W. Duin, D. Ridder, D. M. J. Tax, Classification, Parameter Estimation and State Estimation: An Engineering Approach using MATLAB, John Wiley \& Sons, 2004.

[15] C. Beleites, U. Neugebauer,et al., Sample size planning for classification models. Anal. Chim. Acta, vol. 760, pp. 25-33, 2013. 